\title{
Opioids and the Gastrointestinal Tract - A Case of Narcotic Bowel Syndrome and Literature Review
}

\author{
Adam D Farmer, ${ }^{1,2 *}$ Ella Ferdinand ${ }^{2}$ and Qasim Aziz ${ }^{1}$ \\ ${ }^{1}$ Center for Digestive Diseases, Blizard Institute of Cell and Molecular Science, Wingate Institute of Neurogastroenterology, Barts and the London \\ School of Medicine and Dentistry, Queen Mary University of London, London, UK; and ${ }^{2}$ Department of Gastroenterology, Shrewsbury and \\ Telford NHS Trust, Shropshire, UK
}

The worldwide use of opiates is increasing yet there is little evidence that in long-term, non-cancer patients, they have an efficacious effect on functional outcomes and quality of life measures. Although it seems paradoxical, chronic opiate use may lead to a pro-nociceptive state. Mechanisms for the development of the hyperalgesic state include activation of the opiate bimodal regulatory systems, dynorphin and spinal cord glia. A potential consequence of chronic opiate usage is the development of narcotic bowel syndrome, which is characterized by chronic or intermittent colicky abdominal pain or discomfort that worsens after the narcotic effects of opiates wear off. It is likely that this is an under-recognized diagnosis. We describe here a case of 26-year old female who had visited our institution multiple times with intractable chronic abdominal pain in the context of normal findings on haematological, biochemical, metabolic, endoscopic and radiological investigations. She had been treated with a multitude of opioid agonists with escalating doses. A diagnosis of narcotic bowel syndrome was made. On elective admission her daily analgesic requirements were $150 \mu \mathrm{g} / \mathrm{hr}$ fentanyl, $100 \mathrm{mg}$ oramorph and $400 \mathrm{mg}$ tramadol (equating to $740 \mathrm{mg}$ oral morphine/24 hr). A detoxification regimen was prescribed which included rapid opiate withdrawal couple with the commencement of methadone, lorazepam, clonidine and duloxetine. She was discharged opiate free, with no abdominal pain, 14 days after admission. Clinicians must be aware of narcotic bowel syndrome, which is often erroneously labelled as a functional gastrointestinal disorder, in patients who have been on long-term opiates.

(J Neurogastroenterol Motil 2013;19:94-98)

\section{Key Words}

Analgesics; Gastrointestinal tract; Narcotics; Opioid

\section{Introduction}

Chronic pain represents a considerable worldwide public health concern, consuming considerable healthcare resources.
Opioids are often prescribed for chronic pain but their utility is frequently limited by their gastrointestinal (GI) side effects. For instance, constipation is known to occur in $15-90 \%$ of patients receiving opiates and is known to have a negative impact on health related quality of life (QOL). ${ }^{1}$ Whilst the long-term use of opi-

Received: September 12, 2012 Revised: September 16, 2012 Accepted: September 17, 2012

(c) This is an Open Access article distributed under the terms of the Creative Commons Attribution Non-Commercial License (http://creativecommons. org/licenses/by-nc/3.0) which permits unrestricted non-commercial use, distribution, and reproduction in any medium, provided the original work is properly cited.

*Correspondence: Adam D Farmer, MB, BS, BSc (Hons), PhD, MRCP (UK) Princess Royal Hospital, Apley Castle, Telford, Shropshire TF1 6TF, UK

Financial support: None. Tel: +44-1952641222 (ext. 5644), Fax: +44-7880540584, E-mail: a.farmer@qmul.ac.uk

Conflicts of interest: None.

Author contributions: ADF and EF provided clinical care and wrote the manuscript. QA revised the manuscript for important intellectual content. 
ates in patients with chronic non-cancer pain escalates, evidence suggests that opiates fail to fulfil any of the key outcomes in terms of adequate pain relief, improved QOL or improvements in functional capacity. ${ }^{2,3}$

\section{Case Report}

A 26-year old woman had presented our institution on multiple occasions with a 5 -year history of intractable chronic abdominal pain. Three years prior to admission she had been diagnosed with slow transit constipation, on the basis of a colonic marker study, and had been tried on multiple laxatives. In the absence of any discernable therapeutic benefit she was referred for a tertiary opinion where she underwent anorectal physiology and biofeedback without any changes in her symptoms. Subsequently, she underwent a subtotal colectomy, which increased the frequency of her bowel movement but had no effect on her pain.

She described the pain as being omnipresent, burning in na- ture throughout her abdomen. There were no specific findings on the haematological, biochemical, metabolic, immunological, endoscopic and radiological investigations. Of specific note, her computed tomographical scan of abdomen and pelvis and ileocolonoscopy with histology revealed normal findings in the last 12 months. She was then started on a number of opioid agonists in increasing doses.

The first author decided to electively admit her for further management. At this point her daily analgesic requirements were $150 \mu \mathrm{g} / \mathrm{hr}$ fentanyl, $100 \mathrm{mg}$ oramorph and $400 \mathrm{mg}$ tramadol (equivalent to approximately $740 \mathrm{mg}$ oral morphine/24 hr). A clinical diagnosis of narcotic bowel syndrome (NBS) was made. A detoxification regimen was prescribed which included rapid opiate withdrawal and commencement of methadone, lorazepam, clonidine and duloxetine. She was discharged opiate free, with no abdominal pain, 14 days post admission. At outpatient review for 3 months after discharge, her abdominal pain has not returned and she remains well with no further hospital admissions. She is

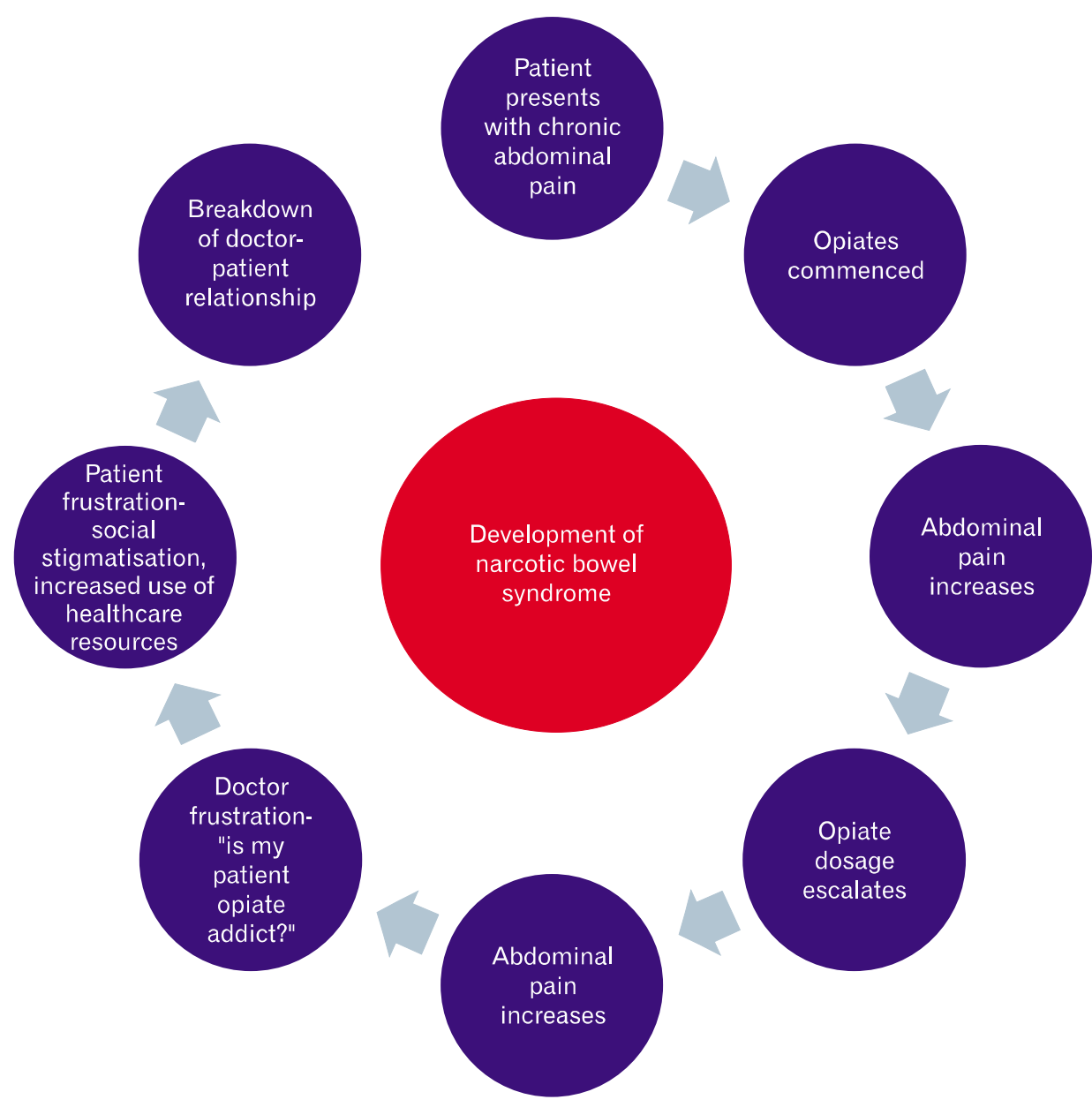

Figure 1. The development of narcotic bowel syndrome. The patient presents with chronic, often abdominal pain and is prescribed with escalating doses of opiates, resulting in no relief in pain. For a number of reasons this can lead to breakdown of the doctor patient relationship. 
under active follow-up. Informed written consent was obtained from the patient.

\section{Discussion}

Paradoxically hyperalgesia can be a consequence of long-term chronic opiate usage. Despite being recognized as a clinical entity over 25 years, it remains as under-recognized side effect amongst clinicians. ${ }^{4}$ NBS is characterized by chronic or intermittent colicky abdominal pain or discomfort that worsens after the narcotic effects of opiates wear off. Over time, the pain free periods become shorter in duration despite increasing doses of opiates. Escalating the opiate dosage only worsens the effect on pain sensitivity by further reducing GI secretion and motility (Fig. 1).

Emerging evidence from a number of published studies has shown that pain is dynamically modulated by central and peripheral factors which can inhibit, as well as facilitate, pain perception. ${ }^{5}$ Chronic opiate use induces neuroplastic changes that paradoxically enhance hyperalgesia and give rise to tolerance. It has been proposed that there are at least 3 mechanisms induced by long-term opiates, thereby leading to this state: bimodal opioid dysregulation, abnormalities in counter-regulatory mechanisms and glial activation. ${ }^{5}$

\section{Bimodal Opioid Regulatory System}

Bimodal refers to both the excitatory and inhibitory modulation of the sensory neuron action by opiates, mediated by $\mathrm{G}_{\mathrm{s}}$ receptor activation at the dorsal root ganglia. Preferential activation of these excitatory pathways may, over time, lead to opiate toleration and pain augmentation. These excitatory effects have been seldom recognized due to the inhibitory effects that opioids have when administered in analgesically active (high) concentrations. Through chronic opiate administration, the $G_{\mathrm{s}}$ receptor becomes super-sensitized resulting in tolerance and ultimately hyperalgesia. ${ }^{6}$ These observations provide rationale for concomitant administration of low dose opiate antagonists, such as methylnaltrexone and alvimopan, in preventing $\mathrm{G}_{\mathrm{s}}$ receptor mediated super-sensitivity thereby enhancing exogenous opioid mediated analgesia at lower doses. ${ }^{7}$

\section{Counter Regulatory Mechanisms}

Dynorphin, an endogenous opiate, when released at the level of the spinal cord increases the release of excitatory neurotransmitters from primary afferents thereby amplifying the afferent signal. Evidence for this pro-nociceptive role comes from ob- servations that dynorphin is increased in chronic pain states and that pain behaviours in animals are attenuated following the administration of dynorphin antagonists. ${ }^{8,9}$ Chronic opiate up regulates dynorphin production at the dorsal horn, inducing further hyperalgesia. Studies blocking this effect have demonstrated that hyperalgesia is reduced, analgesia is restored and opiate tolerance is prevented. ${ }^{10}$

\section{Glial Activation and Opioid Facilitation}

Activation of spinal cord glia is a relatively recently described mechanism that is involved in pain amplification in chronic pain states. Spinal dorsal horn glia, which is considered to be part of the supporting tissue for neurons, can be activated in response to a number of factors including inflammation, infection, opiates, peripheral injury and in response to central signals allowing the possibility of central effects of stress facilitating the pain at a peripheral level. ${ }^{11}$ Exogenous opiates, binding directly to the mu receptor cause the activation of glia with the concomitant release of pro-inflammatory cytokines, to which glial cells express receptors, thereby accelerating the development of hyperalgesia. Glia, in response to the release of inflammatory mediators, may activate neurons through a novel chemokine, known as fractalkine, mediated mechanism. ${ }^{12}$

\section{Management of Narcotic Bowel Syndrome}

To date there is an absence of international consensus with respect to the management of NBS, but central to a successful outcome is the physician-patient relationship. In particular, validation of a patient's symptoms in a supportive environment is, in our opinion, an absolute cornerstone of treatment. For instance, many, if not most, of these patients may have been erroneously diagnosed with a functional GI disorder (FGID) or experienced negative attitudes towards their opiate needs from non-specialist healthcare professionals for many years prior to a definitive diagnosis being made. In this respect, patient education of the pathophysiology of NBS, and therefore the rationale for opiate withdrawal, is a prerequisite step before initiation of treatment. Whereas the preparatory phase for treatment may be undertaken as an outpatient, we advocate that the opiate withdrawal should be started as an in-patient a secondary or tertiary care hospital under the supervision of an experienced specialist.

\section{Treatment modalities}

The specific goals of treatment involve the initiation of treatments that minimize the immediate withdrawal effects of opiates in addition to treating psychological co-morbidities whilst achiev- 


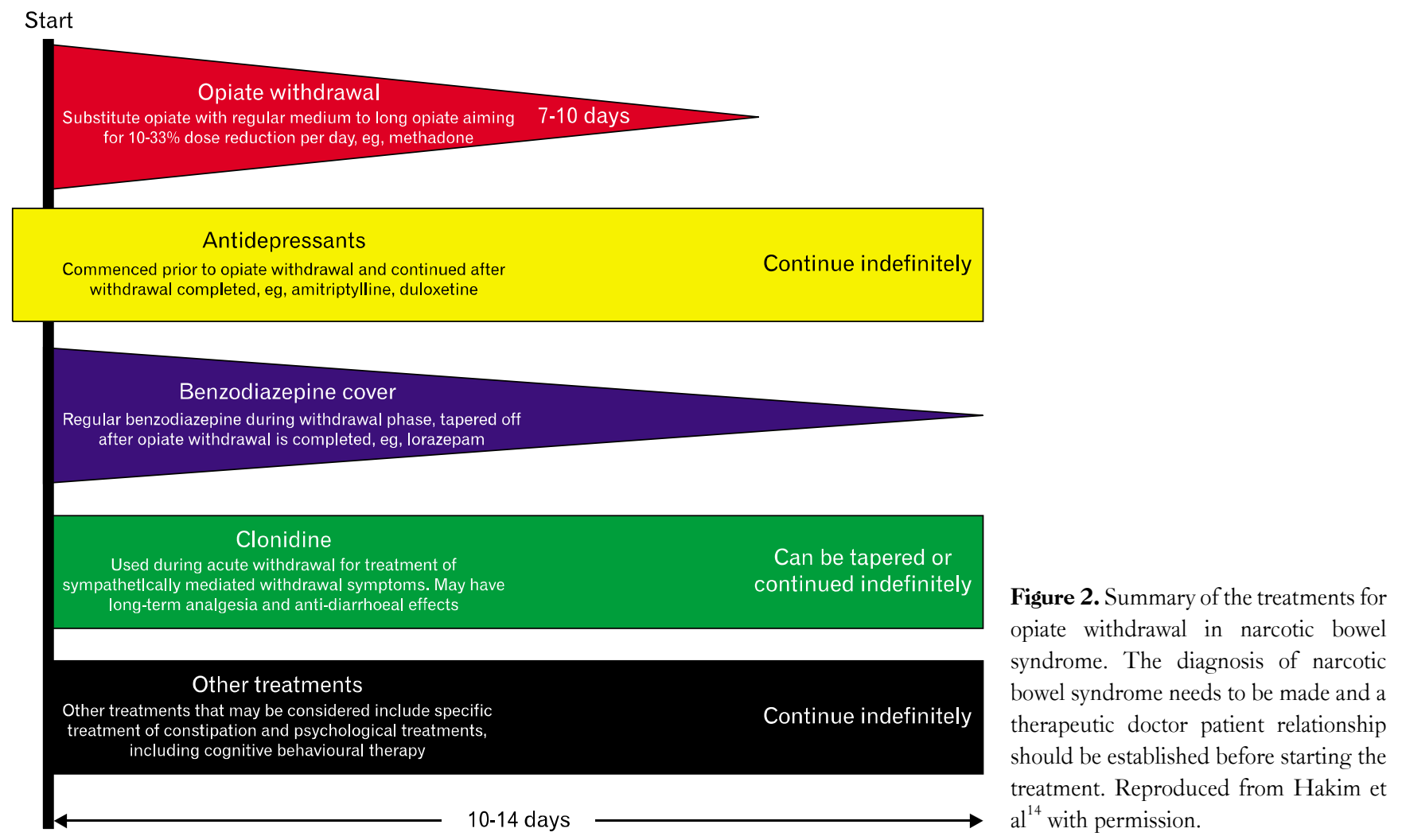

ing pain control for the underlying pathology. The time frame that is needed for a successful opiate withdrawal is extremely variable but it is our experience that the typical period needed for full detoxification is 10-14 days. Grunkemeier et al, ${ }^{5}$ in their excellent article, advocated switching the established opiate for an equivalent dose of a medium to long acting opioid, such as methadone, and then decreasing the dose of this by $10-33 \%$ per day. A divided daily dose should be used to limit large variations in plasma concentrations thereby avoiding rebound pain during trough levels. ${ }^{5}$ However, clinician should be cautious as methadone may systemically accumulate from the fifth day after administration. ${ }^{13}$ When rapid withdrawal regimens are used, patients need to be closely monitored for orthostatic hypotension, syncope, urinary retention or cardiac dysrhythmias. Concomitant medications can be started during the opiate withdrawal phase, for variable periods, to treat coexisting anxiety, psychological co-morbidity and provide long-term central analgesia such as antidepressants, benzodiazepines and clonidine. Figure 2 summarizes a suggested pharmacological approach to opiate withdrawal. ${ }^{14}$ The suggested protocol was useful in our patient, however close liaison with other disciplines, such as the pain management team, could be useful in improving outcomes.
Patients may develop constipation during opiate withdrawal and in the absence of GI obstruction may be treated with laxatives. Although there is no convincing evidence for recommending one particular type of cathartics over another, polyethylene glycol solutions have been shown to be efficacious in the treatment of chronic idiopathic constipation and in our experience are well tolerated. ${ }^{15}$ As discussed earlier, novel mu opioid receptor antagonists, such as methylnaltrexone and alvimopan, may have benefit in the management of opioid induced constipation. A recent reported study evaluated the efficacy of 12 weeks of treatment with sub-cutaneous methylnatrexone in 4 patients with NBS. ${ }^{16}$ It was shown that methylnatrexone had a positive impact on symptoms, although transient pain was problematic following its administration. Long-term psychological support after the initial detoxification is invariably needed. Educating patients in psychological strategies allowing them to manage their chronic pain may improve long-term symptom management and empowers patients to achieve a sense of control over their symptoms.

In conclusion, NBS is a recognised sequelae of chronic opiate administration. Accumulating experimental evidences are providing support to the hypothesis of opiate mediated hyperalgesia. Clinicians must remain mindful of the diagnosis of NBS in pa- 
tients whom have been administered on long-term opiates. Such patients are often erroneously diagnosed - at best as having FGID and at worst as an opiate addict. Further research is now warranted to explore the molecular mechanisms that underlie this paradox, the identification of risk factors for development of this disorder and finally the most efficacious withdrawal regimens.

\section{References}

1. Davis MP. The opioid bowel syndrome: a review of pathophysiology and treatment. J Opioid Manag 2005;1:153-161.

2. Trescot AM, Boswell MV, Atluri SL, et al. Opioid guidelines in the management of chronic non-cancer pain. Pain Physician 2006;9:139.

3. Eriksen J, Sjøgren P, Bruera E, Ekholm O, Rasmussen NK. Critical issues on opioids in chronic non-cancer pain: an epidemiological study. Pain 2006;125:172-179.

4. Sandgren JE, McPhee MS, Greenberger NJ. Narcotic bowel syndrome treated with clonidine. Resolution of abdominal pain and intestinal pseudo-obstruction. Ann Intern Med 1984;101:331-334.

5. Grunkemeier DM, Cassara JE, Dalton CB, Drossman DA. The narcotic bowel syndrome: clinical features, pathophysiology, and management. Clin Gastroenterol Hepatol 2007;5:1126-1139.

6. Crain SM, Shen KF. Antagonists of excitatory opioid receptor functions enhance morphine's analgesic potency and attenuate opioid tolerance/dependence liability. Pain 2000;84:121-131.

7. Lobmaier P, Kornor H, Kunoe N, Bjorndal A. Sustained-release naltrexone for opioid dependence. Cochrane Database Syst Rev 2008;(2):CD006140.

8. Vanderah TW, Gardell LR, Burgess SE, et al. Dynorphin promotes abnormal pain and spinal opioid antinociceptive tolerance. J Neurosci 2000;20:7074-7079.

9. Bian D, Ossipov MH, Ibrahim M, et al. Loss of antiallodynic and antinociceptive spinal/supraspinal morphine synergy in nerve-injured rats: restoration by MK-801 or dynorphin antiserum. Brain Res 1999;831:55-63.

10. Nichols ML, Lopez Y, Ossipov MH, Bian D, Porreca F. Enhancement of the antiallodynic and antinociceptive efficacy of spinal morphine by antisera to dynorphin A (1-13) or MK-801 in a nerve-ligation model of peripheral neuropathy. Pain 1997;69:317-322.

11. Milligan ED, Sloane EM, Watkins LR. Glia in pathological pain: a role for fractalkine. J Neuroimmunol 2008;198:113-120.

12. Watkins LR, Hutchinson MR, Johnston IN, Maier SF. Glia: novel counter-regulators of opioid analgesia. Trends Neurosci 2005;28: 661-669.

13. Nicholson AB. Methadone for cancer pain. Cochrane Database Syst Rev 2007;(4):CD003971.

14. Hakim A, Keer R, Grahame R. Hypermobility, fibromyalgia and chronic pain. London: Churchill Livingstone 2010.

15. Di Palma JA, Cleveland MV, McGowan J, Herrera JL. An open-label study of chronic polyethylene glycol laxative use in chronic constipation. Aliment Pharmacol Ther 2007;25:703-708.

16. Gibson PR, Morrison G. Effects of methylnaltrexone in patients with narcotic bowel syndrome: a pilot observational study. Intern Med 2012;42:907-912. 